\title{
A Dynamical Method for Measuring the Masses of Stars with Transiting Planets
}

\section{Citation}

Loeb, Abraham. 2005. "A Dynamical Method for Measuring the Masses of Stars with Transiting Planets." The Astrophysical Journal 623 (1): L45-48. https://doi.org/10.1086/429873.

\section{Permanent link}

http://nrs.harvard.edu/urn-3:HUL.InstRepos:41393408

\section{Terms of Use}

This article was downloaded from Harvard University's DASH repository, and is made available under the terms and conditions applicable to Other Posted Material, as set forth at http:// nrs.harvard.edu/urn-3:HUL.InstRepos:dash.current.terms-of-use\#LAA

\section{Share Your Story}

The Harvard community has made this article openly available.

Please share how this access benefits you. Submit a story.

\section{Accessibility}


Draft Version June 15, 2018

Preprint typeset using LATEX style emulateapj v. 6/22/04

\title{
A DYNAMICAL METHOD FOR MEASURING MASSES OF STARS WITH TRANSITING PLANETS
}

\author{
ABraham Loeb \\ Astronomy Department, Harvard University, 60 Garden Street, Cambridge, MA 02138; aloeb@cfa.harvard.edu
}

Draft version June 15, 2018

\begin{abstract}
As a planet transits the face of a star, it accelerates along the line-of-sight. The changing delay in the propagation of photons produces an apparent deceleration of the planet across the sky throughout the transit. This persistent transverse deceleration breaks the time-reversal symmetry in the transit lightcurve of a spherical planet in a circular orbit around a perfectly symmetric star. For "hot Jupiter" systems, ingress advances at a higher rate than egress by a fraction $\sim 10^{-4}-10^{-3}$. Forthcoming space telescopes such as Kepler or COROT will reach the sensitivity required to detect this asymmetry. The scaling of the fractional asymmetry with stellar mass $M_{\star}$ and planetary orbital radius $a$ as $\propto M_{\star} / a^{2}$ is different from that of the orbital period as $\propto\left(M_{\star} / a^{3}\right)^{-1 / 2}$. Therefore, this effect constitutes a new method for a purely dynamical determination of the mass of the star, which is currently inferred indirectly with theoretical uncertainties based on spectral modeling. Radial velocity data for the reflex motion of the star can then be used to determine the planet's mass. Although orbital eccentricity could introduce a larger asymmetry than the light propagation delay, the eccentricity is expected to decay by tidal dissipation to negligible values for a close-in planet with no perturbing third body. Future detection of the eclipse of a planet's emission by its star could be used to measure the light propagation delay across the orbital diameter, $46.7\left(a / 7 \times 10^{11} \mathrm{~cm}\right)$ seconds, and also determine the stellar mass from the orbital period.
\end{abstract}

Subject headings: planetary systems, techniques: photometric

\section{INTRODUCTION}

The population of known extrasolar planets which transit the face of their parent stars has been growing steadily in recent years. It currently includes HD 209458b (Charbonneau et al. 2000), OGLE-TR56b (Torres et al. 2004), OGLE-TR-113b (Konacki et al. 2004a), OGLE-TR-132b (Moutou et al. 2004), TrES-1 (Alonso et al. 2004), OGLE-TR-111b (Pont et al. 2004) and OGLE-TR10b (Konacki et al. 2004b). For HD 209458b , the transit lightcurve was observed with the Hubble Space Telescope (HST) to be symmetric around its centroid to an exquisite photometric precision of $\sim 10^{-4}$ magnitude per data point for a few hundred data points tracing both ingress and egress (Brown et al. 2001).

In this Letter we show that transit lightcurves must have a time-reversal asymmetry even if the star and the planet are perfectly symmetric and the orbit is circular. The asymmetry originates from the persistent acceleration of the planet towards the star during the transit. For a circular orbit, the planet moves towards the observer at the beginning of the transit (ingress) and away from the observer at its end (egress). This net acceleration introduces a change in the relative rate by which ingress and egress advance in the observer's frame of reference. The effect simply follows from the unsteady change in the propagation delay of photons which distorts the transformation of time between the planet and the observer. The variation rate of the delay is changing most rapidly at the middle of the transit when the full Newtonian acceleration vector points straight along the line-of-sight. In the following sections, we derive the magnitude of the resulting lightcurve asymmetry $(\S 2)$ and discuss its implications $(\S 3)$.

\section{APPARENT TRANSVERSE DECELERATION DUE TO PROPAGATION DELAY OF PHOTONS}

The observed arrival time of photons $t_{\mathrm{obs}}$ is given by the time they left the planet $t$ plus the propagation delay over the distance of the planet $D$,

$$
t_{\mathrm{obs}}=t+D / c \text {. }
$$

The $t$-derivative of equation (1) gives

$$
d t_{\mathrm{obs}}=d t\left(1+\frac{v_{\|}}{c}\right)
$$

where $v_{\|}(t)=d D / d t$ is the velocity of the planet along the line-of-sight. Since $v_{\|}$changes from negative to positive during the transit of a planet in a circular orbit, it is now obvious that ingress would advance at a faster rate (i.e. a shorter $d t_{\text {obs }}$ per $d t$ interval) than egress. Figure 1 illustrates schematically this generic behaviour. Note that equation (2) is accurate to all orders in $|\mathbf{v} / c|$ but we will keep only first order terms in subsequent derivations. Even though the planet is not emitting the observed photons, the timing of its occultation is dictated by the instant at which stellar photons graze its outer surface; this timing is distorted by the same propagation delay effect that would exist if the grazing photons were emitted by the planet itself rather than the star since the propagation history of the photons before they graze the planet's boundary is irrelevant. The transverse spatial coordinates of the planet orbit, $\mathbf{x}_{\perp}$, are the same in the observer and planet reference frames. The apparent transverse velocity of the planet is therefore different from its actual transverse velocity, $\mathbf{v}_{\perp}=\left(d \mathbf{x}_{\perp} / d t\right)$ (Rvbicki \& Lightman 1979),

$$
\mathbf{v}_{\perp, \mathrm{obs}} \equiv \frac{d \mathbf{x}_{\perp}}{d t_{\mathrm{obs}}}=\left(\frac{d t}{d t_{\mathrm{obs}}}\right) \frac{d \mathbf{x}_{\perp}}{d t} \approx\left(1-\frac{v_{\|}}{c}\right) \mathbf{v}_{\perp},
$$



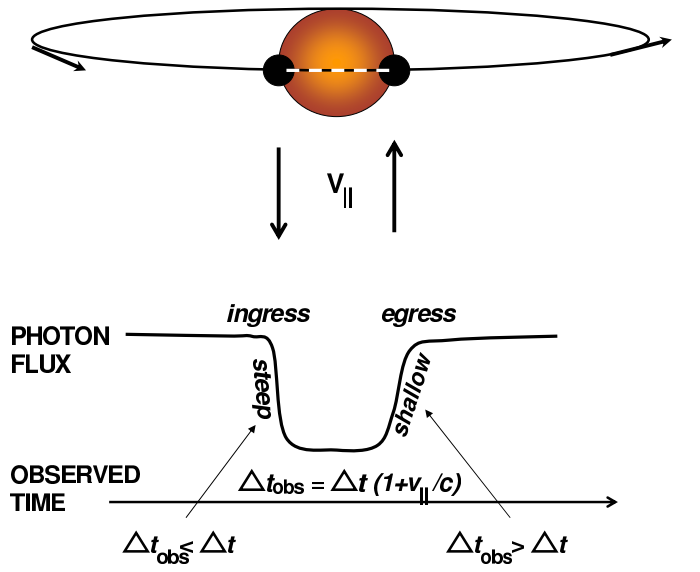

FIG. 1.- Schematic illustration of the propagation delay effect. The transiting planet is moving towards the observer $\left(v_{\|}<0\right)$ during ingress and away from the observer $\left(v_{\|}>0\right)$ during egress. As a result, the observed time interval of partial eclipse is shorter at ingress relative to egress (see Eq. 2). This breaks the time reversal symmetry of the observed lightcurve and introduces a fractional difference of $\delta v_{\|} / c$ in the temporal slope of the lightcurve between ingress and egress, where $\delta v_{\|}=\dot{v}_{\|} \tau$ is the net gain in the planet's line-of-sight velocity over the transit duration $\tau$. For close-in planets, the fractional asymmetry in the slopes is of order $\delta \sim 10^{-4}-10^{-3}$ (see Eq. [7).

where the transverse position vector $\mathbf{x}_{\perp}$ corresponds to angular coordinates on the sky times the distance to the planetary system. Throughout our discussion, the terms parallel $(\|)$ or transverse $(\perp)$ are relative to the line-ofsight axis that starts at the observer and goes through the center of the stellar image. We focus on the time when the occulting planet crosses this spatial point of symmetry.

Taking the $t_{\mathrm{obs}}$-derivative of both sides of equation (3) and keeping terms to leading-order in $|\mathbf{v} / c|$, we get two new contributions to the difference between the observed and Keplerian values of the transverse acceleration of the planet across the sky (Loeb 2003),

$$
\dot{\mathbf{v}}_{\perp, \mathrm{obs}}=\dot{\mathbf{v}}_{\perp}-\frac{2 v_{\|}}{c} \dot{\mathbf{v}}_{\perp}-\frac{\mathbf{v}_{\perp}}{c} \dot{v}_{\|},
$$

where $\dot{\mathbf{v}}_{\text {obs }} \equiv\left(d^{2} \mathbf{x} / d t_{\text {obs }}^{2}\right)$ is the observed acceleration and $\dot{\mathbf{v}} \equiv\left(d^{2} \mathbf{x} / d t^{2}\right)$ is the actual Keplerian acceleration of the planet. The last term on the right-hand-side of equation (4) implies that the apparent transverse acceleration of the planet gets a contribution from its Keplerian acceleration along the line-of-sight, $\dot{v}_{\|}$. This term is the source of our effect.

For simplicity, we assume that the orbital plane is viewed edge-on by the observer; this geometry is exact for transits that cross the center of the star ("central transits") and is a very good approximation more generally as long as the orbital radius is much larger than the radius of the star. For a circular planetary orbit, Newtonian dynamics implies no transverse acceleration of the planet at the spatial center of symmetry of the transit where

$$
\dot{\mathbf{v}}_{\perp}=0 ; v_{\perp}^{2}=\frac{G M_{\star}}{a} ; \dot{v}_{\|}=\frac{G M_{\star}}{a^{2}} ; v_{\|}=0 .
$$

Here $M_{\star}$ is the stellar mass and $a$ is the planet's orbital radius. However, equation (4) implies that at the same time there would be an apparent transverse deceleration of the planet in the observer's frame of reference

$$
\dot{\mathbf{v}}_{\perp, \mathrm{obs}}=-\frac{\mathbf{v}_{\perp}}{c} \dot{v}_{\|}=-\frac{\mathbf{v}_{\perp}}{\left|\mathbf{v}_{\perp}\right|} \frac{\left(G M_{\star}\right)^{3 / 2}}{a^{5 / 2} c},
$$

which is directed opposite to $\mathbf{v}_{\perp}$. This persistent apparent deceleration implies that the planet would cross ingress and egress at different apparent rates. Thus, even if the stellar image is perfectly symmetric, the orbit is circular and the planet is perfectly spherical, there is an inherent asymmetry in the transit lightcurve due to the nearly steady value of $\dot{v}_{\|}=G M_{\star} / a^{2}$ during the transit. Newtonian dynamics alone predicts a non-vanishing $\dot{\mathbf{v}}_{\perp}$ as soon as the planet moves away from the transit center, but for a circular orbit this deviation would maintain the time-reversal symmetry between ingress and egress.

The fractional asymmetry in the transit lightcurve is of order the fractional change in $v_{\perp, \text { obs }}$ over the transit duration, since the rates by which ingress and egress proceed are proportional to $v_{\perp, \text { obs }}$. For a total transit duration $\tau$ and an orbital period $T=2 \pi a / v_{\perp} \gg \tau$, the slope of the initial drop and final rise in the transit lightcurve will differ by a fractional amplitude

$$
\begin{aligned}
\delta & \equiv \frac{\delta v_{\perp, \mathrm{obs}}}{v_{\perp, \mathrm{obs}}}=\left|\frac{\dot{\mathbf{v}}_{\perp, \mathrm{obs}} \tau}{\mathbf{v}_{\perp, \mathrm{obs}}}\right|=\frac{\dot{v}_{\|} \tau}{c}=\left(\frac{v_{\perp}}{c}\right)\left(\frac{2 \pi \tau}{T}\right) \\
& =1.078 \times 10^{-4}\left(\frac{M_{\star}}{1.1 M_{\odot}}\right)\left(\frac{a}{7 \times 10^{11} \mathrm{~cm}}\right)^{-2}\left(\frac{\tau}{3 \mathrm{hr}}\right) .
\end{aligned}
$$

For a "hot Jupiter" like HD 209458b, the ingress phase $\left(v_{\|}<0\right)$ would proceed at a rate that is higher by fractional amplitude of $\sim 10^{-4}$ than the egress phase $\left(v_{\|}>0\right)$. Planets that are closer in by a factor of a few could produce an asymmetry of up to $\delta \sim 10^{-3}$; OGLETR-56b for which $a=3.5 \times 10^{11} \mathrm{~cm}$ provides $\delta=3 \times 10^{-4}$ [see Gaudi et al. (2005) for a compilation of all known transit systems and the prospects for detecting others].

The observed time $t_{\mathrm{obs}}$ is a slightly distorted version of the time axis $t$ along which the lightcurve is symmetric. We may write $t_{\mathrm{obs}}=t(1+\epsilon)$, where $\epsilon(t)=(t / 2 \tau) \delta$ and we shifted $t=0$ to be at the transit centroid. The observed photon flux, $F\left(t_{\text {obs }}\right)$, corresponds to the flux at an undistorted time $t \approx t_{\mathrm{obs}}[1-\epsilon(t)]$, while $F\left(-t_{\mathrm{obs}}\right)$ corresponds to $t \approx-t_{\mathrm{obs}}[1-\epsilon(-t)]=-t_{\mathrm{obs}}[1+\epsilon(t)]$. For small deviations, we may expand the photon flux as a function of time to leading order, $F(t+\Delta t) \approx F(t)+\left.(d F / d t)\right|_{t} \Delta t$. Since $\epsilon(t)=-\epsilon(-t), F(t)=F(-t)$, and $\left.(d F / d t)\right|_{t}=$ $-\left.(d F / d t)\right|_{-t}$, the time-dependent photometric asymmetry of the lightcurve is given by

$$
\begin{aligned}
\Delta_{F}\left(t_{\mathrm{obs}}\right) & \equiv \frac{F\left(t_{\mathrm{obs}}\right)-F\left(-t_{\mathrm{obs}}\right)}{F\left(t_{\mathrm{obs}}\right)}= \\
& =-\left(\frac{d \ln F}{d \ln t_{\mathrm{obs}}}\right)_{t_{\mathrm{obs}}} \times\left(\frac{t_{\mathrm{obs}}}{\tau}\right) \delta,
\end{aligned}
$$

where we have set $t_{\mathrm{obs}}=0$ at the transit centroid (for which $d F / d t_{\mathrm{obs}}=0$ ) and kept terms to leading order in 


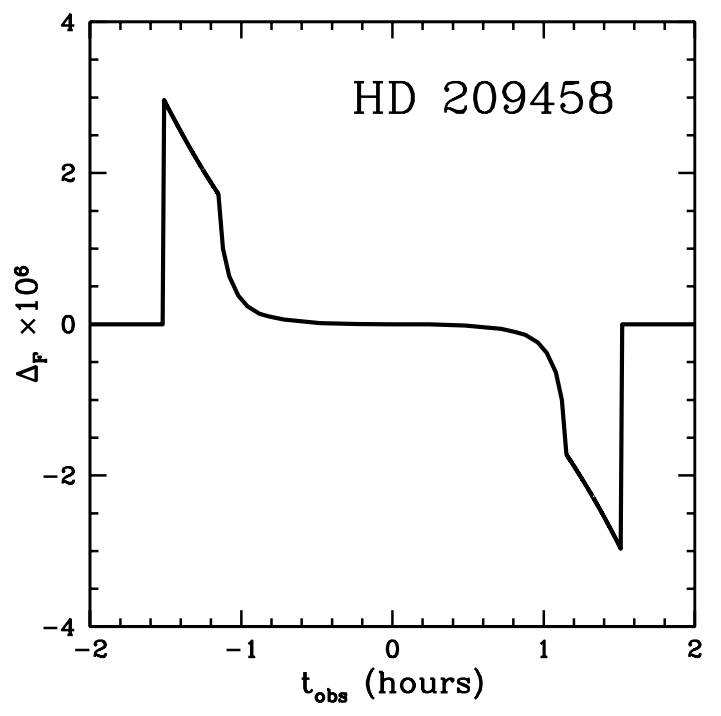

FIG. 2.- Predicted fractional deviation from time-reversal symmetry, $\Delta_{F}\left(t_{\mathrm{obs}}\right)$, for the observed transit lightcurve of HD 209458b (Brown et al. 2001), based on Eqs. (7) and (8). The vertical axis is proportional to $\left(M_{\star} / 1.1 M_{\odot}\right)\left(a / 7 \times 10^{11} \mathrm{~cm}\right)^{-2}$. The four breaks in the curve result from the four points of contact between the projected planet boundary and the limb of the star.

$|\epsilon| \ll 1$. Given a preliminary transit lightcurve, $F\left(t_{\mathrm{obs}}\right)$, and an approximate knowledge of the system parameters, it is possible to predict the lightcurve asymmetry under the assumption of a circular orbit. Figure 2 shows the expected $\Delta_{F}\left(t_{\mathrm{obs}}\right)$ for HD 209458b based on the lightcurve data in Brown et al. (2001). For a transit depth of $2 \times 10^{-2}$ magnitudes produced by a close-in planet, the net photometric asymmetry would typically amount to a few percent of $\delta$ or equivalently to a peak value of $\left|\Delta_{F}\right|$ in the range of $(0.1-1) \times 10^{-5}$. The photometric sensitivity of $\sim 10^{-4}$ magnitudes per data point achieved with $H S T$ for HD 209458b (Brown et al. 2001) provides a net sensitivity to a time-reversal asymmetry of $\left|\Delta_{F}\right| \sim 10^{-5}\left(N / 10^{2}\right)^{-1 / 2}$, where $N \sim 10^{2}$ is the total number of independent data points during ingress and egress. Our effect appears to be on the borderline of being detectable with existing techniques. A statistically significant $(\gtrsim 3 \sigma)$ detection of the asymmetry in equation (7) for HD 209458b would require an additional order of magnitude improvement in sensitivity, which is achievable with future space missions such as Kepler ${ }^{1}$ or $C O R O T^{2}$ and only marginally with the existing instrument $\operatorname{MOST}^{3}$. A simple way to reach the required sensitivity would be to maintain the existing photometric precision per data point and increase the number of data points from $\sim 10^{2}$ up to $\sim 10^{4}$ (e.g. by increasing the number of HD 209458b transits from the 4 observed by $H S T$ to $\sim 400$ ) over a period of several years.

The orbital period scales as $\propto\left(M_{\star} / a^{3}\right)^{-1 / 2}$ while the fractional asymmetry in equation (7) scales as $\propto M_{\star} / a^{2}$. Thus, detection of the lightcurve asymmetry would allow to determine $M_{\star}$ and $a$ separately. Additional data on the reflex motion of the star would then provide the

\footnotetext{
${ }^{1}$ http://www.kepler.arc.nasa.gov/summary.html

2 http://serweb.oamp.fr/projets/corot/

3 http://www.astro.ubc.ca/MOST/
}

planet mass, $M_{\mathrm{P}}$, based on dynamical information alone. Currently, the stellar mass, $M_{\star}$, is inferred indirectly based on theoretical fitting of the stellar spectrum which is subject to modeling uncertainties at the level of $\gtrsim 10 \%$ [see Fig. 3 in Alonso et al. (2004)].

If the planetary orbit is nearly circular but has a finite eccentricity, $e \ll 1$, then it is easy to show that at the transit center

$$
\dot{v}_{\perp}=-e \sin \Psi \frac{G M_{\star}}{a^{2}},
$$

to leading order in $e$, where $\Psi$ is the angle between the shortest radius vector of the orbital ellipse (along the apsidal line) and the line to the observer. As long as $|e \sin \Psi|<\left|v_{\perp} / c\right| \lesssim 10^{-3}$, the eccentricity effect is smaller than the propagation delay effect in equation (6). In the absence of perturbers, any initial orbital eccentricity is expected to decay exponentially through tidal dissipation on an e-folding timescale (Goldreich \& Soter 1966)

$$
\begin{aligned}
& t_{\text {circ }}=\frac{e}{\dot{e}}=\left(\frac{4 T Q_{\mathrm{P}}}{63 \times 2 \pi}\right)\left(\frac{M_{\mathrm{P}}}{M_{\star}}\right)\left(\frac{a}{R_{\mathrm{P}}}\right)^{5}= \\
= & 1.4 \times 10^{-2}\left(\frac{Q_{\mathrm{P}}}{10^{5}}\right)\left(\frac{M_{\mathrm{P}}}{6 \times 10^{-4} M_{\star}}\right)\left(\frac{a}{75 R_{\mathrm{P}}}\right)^{5} \mathrm{Gyr},
\end{aligned}
$$

where the quality parameter $Q_{\mathrm{P}}\left(\sim 10^{5}\right.$ for Jupiter $)$ is inversely proportional to the dissipation rate in the planet's interior (Ioannou \& Lindzen 1993; Ogilvie \& Lin 2004), and $R_{\mathrm{P}}$ is the planet's radius. Aside from the unknown $Q_{\mathrm{P}}$, the parameter values in equations (7) and (10) were chosen to match HD 209458b . Since the orbital circularization timescale is shorter by up to two orders of magnitude than the age of planetary systems such as HD 209458 [ 5 Gyr, see Mazeh et al. (2000) and Codv \& Sasselov (2002)], the eccentricity-driven asymmetry is expected to diminish for a close-in planet unless a third body pumps its orbital eccentricity. Incidentally, such a perturber was hypothesized as the driver of the inflated radius of HD 209458b (Bodenheimer et al. 2003) but its existence has not been demonstrated yet.

For close-in planets such as HD 209458b, our effect is larger than a different source of lightcurve asymmetry that was already discussed in the literature, namely the planet's obliquity (Hui \& Seager 2002b). This is because close-in planets are expected to rotate slowly and possess a small projected obliquity as a result of strong tidal locking with their orbital revolution. Their rotation axis is expected to be normal to their orbital plane which is viewed nearly edge-on [in the case of HD $209458 \mathrm{~b}$, the orbital inclination angle is $i=86 .^{\circ} 1 \pm 1 .{ }^{\circ} 6$ (Mazeh et al. 2000)]. Another source of asymmetry is rotation of the star. Typical projected rotation speeds of $v_{\text {rot }} \sin i \sim 4 \mathrm{~km} \mathrm{~s}^{-1}$ (Mazeh et al. 2000; Queloz et al. 2000 ) would produce a Doppler offset (Loeb \& Gaudi 2003 ) between the flux emitted by the approaching and receding sides of the star of order $\sim\left(v_{\text {rot }} / c\right)=1.3 \times 10^{-5}$ [the photometric analog of the Rossiter-McLaughlin effect; see Queloz et al. (2000); Ohta et al. (2004)], or an occultation contrast of $\sim 10^{-7}$ magnitudes which is much smaller than the effect considered here. If the stellar rotation axis is significantly misaligned with the normal of the orbital plane and the transit is not central, then the rotation-induced oblateness of the stellar image could 
generate a fractional ( $\delta$-equivalent) asymmetry of less than $\frac{1}{2}\left(v_{\text {rot }}^{2} R_{\star} / G M_{\star}\right) \sim 5 \times 10^{-5}$, which is again well below our effect for HD 209458b where the misalignment angle must be small (Queloz et al. 2000).

The propagation delay relation between $t_{\mathrm{obs}}$ and $t$ in equation (11) can be easily incorporated into a computer program that searches for the best-fit Keplerian orbit under the constraints of a given data set. The delaycorrected Keplerian fit would provide new dynamical constraints on the planetary system. Such a fit would involve the same number of free parameters as the standard Keplerian fit.

\section{DISCUSSION}

We have shown that the propagation delay of light introduces an apparent transverse deceleration of a planet on the sky during its transit across the face of its parent star (Eq. 6). This persistent deceleration breaks the time-reversal symmetry of the transit lightcurve for a spherical planet in a circular orbit around a spherical star. Throughout the transit, the planet velocity along the line-of-sight, $v_{\|}$, is changing at a nearly steady rate, $G M_{\star} / a^{2}$. This produces a steady change in the transformation of time to the observer's frame (see Eq. 22. The net change in $v_{\|}$between ingress and egress, $\delta v_{\|}=\dot{v}_{\|} \tau$, introduces a fractional difference of magnitude $\delta=\delta v_{\|} / c$ in the slopes of their lightcurves. It is possible to search for this difference in slopes by folding the lightcurve over its centroid. For close-in planets, ingress should typically proceed at a rate that is faster by a fractional amplitude $\delta \sim 10^{-4}-10^{-3}$ than egress (see Eq. 7). This level of asymmetry will be detectable with forthcoming space telescopes such as COROT or Kepler (Borucki et al. 2003), which are scheduled for launch within 2-3 years. Because this asymmetry has a unique scaling with stellar mass and orbital radius $\left(\propto M_{\star} / a^{2}\right)$, its detection together with the reflex motion of the star will allow to determine the star and planet masses as well as the orbital radius using purely dynamical data. This method evades the theoretical uncertainties inherent in the existing approach for determining stellar masses based on modeling of spectroscopic data [e.g. Codv \& Sasselov (2002)].

Orbital eccentricity could induce a stronger asymmetry in the lightcurve but is expected to decay exponentially to negligible levels through tidal dissipation for close-in planets like HD 209458b , unless it is being pumped by the gravitational perturbation of another planet.

Similarly to other transit timing residuals (Miralda-Escudé 2002; Holman \& Murrav 2004; Agol et al. 2004), our effect would be contaminated by noise from inhomogeneities on the face of the star and would compete against other small effects involving asymmetries from the oblateness of the planet (Hui \& Seager 2002a b) or the rotation of the star. Additional special-relativistic or general-relativistic effects are of the order of $\sim(v / c)^{2}$ or $\sim \phi / c^{2}$, or smaller, where $\phi$ is the gravitational potential produced by the star $\left(\phi \sim v^{2}\right)$; these corrections are orders of magnitude smaller than the propagation delay effect discussed here.

Finally, we note that a change of the opposite sign in $v_{\|}$occurs when the planet goes behind the star. In this case, ingress would be slower than egress. There is no net change in the orbital period over a full closed orbit. However when the planet enters its own (secondary) eclipse by the star, the photons it emits will be delayed relative to primary eclipse (the transit) by the difference in emission times plus the light travel time across the orbital diameter, as implied by equation (11). For a circular orbit, the time interval between the centroids of the primary and secondary eclipses would be longer by $\delta T_{1 / 2}=2 a / c$ than half of the full orbital period. Future detection of a planet's infrared emission would then allow to determine the orbital radius from the light propagation delay of $\delta T_{1 / 2}=46.7\left(a / 7 \times 10^{11} \mathrm{~cm}\right)$ seconds. The full orbital period $T$ would then yield the stellar mass through $M_{\star}=a^{3} /\left[G(T / 2 \pi)^{2}\right]$. An eccentricity could change the lightcurve history of the illuminated planet and in particular make the time from primary eclipse to secondary eclipse different from the time back to primary eclipse.

I thank David Charbonneau, Scott Gaudi, Matt Holman, Dimitar Sasselov, George Rybicki, and Josh Winn for helpful discussions. This work was supported in part by NASA grant NAG 5-13292 and NSF grants AST0071019 and AST-0204514.

\section{REFERENCES}

Agol, E., Steffen, J., Sari, R., \& Clarkson, W. 2004, MNRAS, submitted; astro-ph/0412032

Alonso, R., et al. 2004, ApJ, 613, L153

Bodenheimer, P., Laughlin, G., \& Lin, D. N. C. 2003, ApJ, 592, 555

Borucki, W. J., et al. 2003, in Future EUV/UV and Visible Space Astrophysics Missions and Instrumentation, Ed. J. C, Blades \& O. H. W. Siegmund, Proc. of SPIE, Volume 4854, pp. 129-140

Bouchy, F., Pont, F., Santos, N. C., Melo, C., Mayor, M., Queloz, D., \& Udry, S. 2004, A\&A, 421, L13

Brown, T. M., Charbonneau, D., Gilliland, R. L., Noyes, R. W., \& Burrows, A. 2001, ApJ, 552, 699

Charbonneau, D., Brown, T. M., Latham, D. W., \& Mayor, M. 2000, ApJ, 529, L45

Cody, A. M., \& Sasselov, D. D. 2002, ApJ, 569, 451

Gaudi, S. , Seager, S., \& Mallen-Ornelas, G. 2005, ApJ, in press; astro-ph/0409443

Goldreich, P., \& Soter, S. 1966, Icarus, 5, 375

Holman, M., \& Murray, N. W. 2004, Science, submitted; astro-ph/0412028

Hui, L., \& Seager, S. 2002a, ApJ, 572, 540 2002b, ApJ, 574, 1004

Ioannou, P. J., \& Lindzen, R. S. 1993, ApJ, 406, 266

Konacki, M. et al. 2004a, ApJ, 609, L37

Konacki, M.. Torres, G., Sasselov, D., \& Jha, S. 2004b, astro-ph/0412400

Loeb, A. 2003, astro-ph/0309716

Loeb, A., \& Gaudi, B. S. 2003, ApJ, 588, L117

Mazeh, T., et al. 2000, ApJ, 532, L55

Miralda-Escudé, J. 2002, ApJ, 564, 1019

Moutou, C., Pont, F., Bouchy, F., \& Mayor, M. 2004, A \& A, in press; astro-ph/0407635

Ogilvie, G. 1., \& Lin, D. N. C. 2004, ApJ, 610, 477

Ohta, Y., Taruya, A., \& Suto, Y. 2004, astro-ph/0410499

Pont, F., Bouchy, F., Queloz, D., Santos, N. C., Melo, C., Mayor, M., \& Udry, S. 2004, A \& A, 426, L15

Queloz, D., Eggenberger, A., Mayor, M., Perrier, C., Beuzit, J. L., Naef, D., Sivan, J. P., \& Udry, S. 2000, A\&A, 359, L13

Rybicki, G. B. \& Lightman, A. P. Radiative Processes in Astrophysics, (New York: Wiley, 1979), see problem 4.7a on p. 151

Torres. G., Konacki, M., Sasselov, D., Jha, S. 2004, ApJ, 609, 1071 